\title{
Thomson Scattering Diagnostics in the Plasma of an Ion Thruster
}

\author{
By Naoji YAmAmoto ${ }^{1)}$, Yoshiaki HiraOKA ${ }^{1)}$, Kensaku SugitA ${ }^{1)}$, Tomoaki KuritA ${ }^{1)}$, Kentaro Tomita ${ }^{2)}$, \\ Kiichiro UCHINO ${ }^{2)}$ and Hideki NAKASHIMA ${ }^{1)}$ \\ 1) Department of Advanced Energy Engineering Science, Kyushu University, Fukuoka, Japan \\ ${ }^{2)}$ Department of Applied Science for Electronics and Materials, Kyushu University, Fukuoka, Japan
}

(Received June 18th, 2011)

\begin{abstract}
In order to validate the grid erosion evaluation code for the lifetime validation of ion thrusters, the electron number density/temperature in the vicinity of a screen gird in a $30 \mathrm{~W}$ class microwave discharge ion thruster were measured by means of laser Thomson scattering (LTS) technique. A photon counting method and a triple grating spectrometer were used against a small Thomson scattering signal and a strong stray laser light. Observed Thomson scattering spectrum tells that the electron energy distribution function was Maxwellian. From this spectrum and the Rayleigh scattering calibration using nitrogen gas, electron number density and electron temperature were calculated to be $(3.8 \pm 0.2) \times 10^{17} \mathrm{~m}^{-3}$ and $6.2 \pm 0.1 \mathrm{eV}$, respectively at incident microwave power of $8 \mathrm{~W}$ and krypton mass flow rate of $6.2 \mu \mathrm{g} / \mathrm{s}$. The ion saturation currents estimated from the LTS measurement are in good agreement with ion beam currents through the screen grid for several conditions. These results show that LTS technique is a useful non-intrusive tool for measuring plasma property in the vicinity of the screen grid.
\end{abstract}

Key Words: Ion Thruster, Laser Thomson Scattering Technique, Non-intrusive Plasma Property Measurement

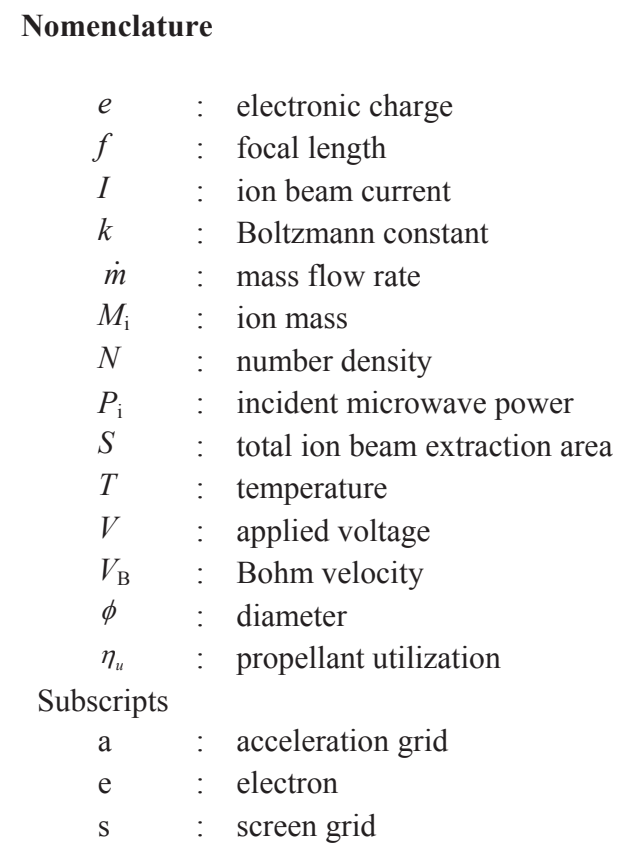

\section{Introduction}

Measurement of plasma property in the vicinity of the screen grid is essential for the development of an ion thruster. Since plasma properties, electron number density and electron energy distribution function (EEDF) are useful for designing a grid system. Furthermore, this information will play an important role in the validation of numerical models for lifetime estimation ${ }^{1)}$. There have been many studies to measure plasma property inside the discharge chamber ${ }^{2}$ and these results contributed to the development of ion thrusters. However, it has been difficult to measure the plasma properties in the vicinity of the screen grid without disturbance by means of intrusive method, such as electrostatic probes. So, non-intrusive method is needed for the measurement of the plasma property in this region.

Therefore, the aim of this study is to measure plasma property in the vicinity of the screen grid by laser Thomson scattering (LTS $)^{3)}$ technique. LTS is a nonintrusive method for a measurement of plasma properties. In the incoherent regime, the scattered spectrum reflects the Doppler motion of individual electrons, and the scattered intensity is proportional to electron number density ${ }^{4}$. This method was developed to measure plasma properties in high temperature plasma having $N_{\mathrm{e}}>10^{19} \mathrm{~m}^{-3}$. During the last decade, its applicability has been extended to lower density plasma, with densities of less than $10^{16} \mathrm{~m}^{-3}$, by a signal accumulation technique ${ }^{5)}$. This technique allows us to apply LTS to the plasma produced in the discharge chamber of ion thrashers.

\section{Experimental Equipment}

\subsection{Microwave discharge ion thruster}

The cross section of a $30 \mathrm{~W}$ class miniature microwave discharge ion thruster is shown in Fig. 1. The inner diameter of the discharge chamber is $21 \mathrm{~mm}$ and the size of the thruster is $50 \mathrm{~mm} \times 50 \mathrm{~mm} \times 30 \mathrm{~mm}$. The ion source consists of an antenna and a magnetic circuit, which is made up of several samarium cobalt ( $\mathrm{Sm}-\mathrm{Co})$ permanent magnets and iron yokes. The magnetic field strength inside the discharge chamber can be changed by changing the number of the permanent magnets. The magnetic mirrors are located at the tip of a front yoke and 
the tip of a central yoke.

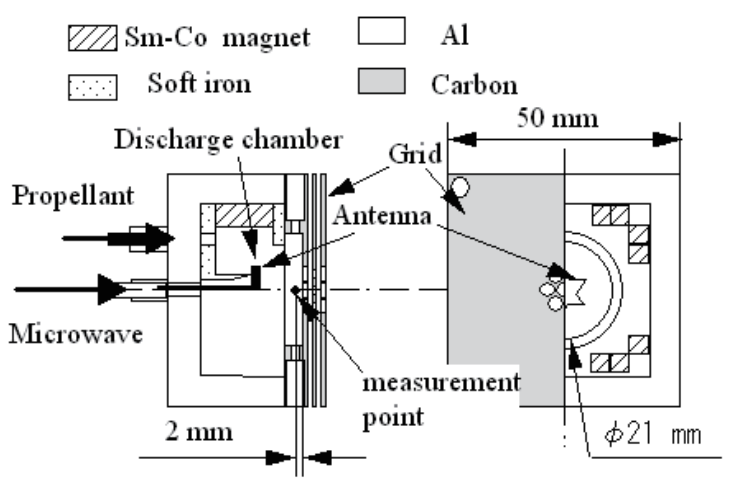

Fig. 1. Cross section of a miniature microwave discharge ion thruster developed at Kyushu University.

Table 1. Grid parameters.

\begin{tabular}{cccc}
\hline \hline Parameter & Screen & Accel. & Decel. \\
\hline Hole diameter, mm & 3.0 & 1.8 & 3.0 \\
Potential, V & 1200 & -200 & 0 \\
Thickness, mm & 1.0 & \\
Hole pitch, mm & 3.50 & \\
Material & Pyrolytic carbon \\
Grid gap, mm & 0.5 \\
Number of holes & 7 \\
\hline \hline
\end{tabular}

Microwave power at $2.45 \mathrm{GHz}$ is fed through a coaxial line and into the antenna. A DC block with a loss of $0.43 \mathrm{~dB}$ at $2.45 \mathrm{GHz}$ was inserted to protect the microwave amplifier. A star-shape antenna is used, since it showed good performance in previous studies ${ }^{6}$. The antenna is inscribed in a $9 \mathrm{~mm}$ diameter circle and is made of molybdenum. The thickness of the antenna is $1 \mathrm{~mm}$. Flat square grids were used to extract the ion beam. The geometric parameters are shown in Table 1. This geometry was designed using a numerical analysis code developed by Arakawa et $\mathrm{al}^{7}$. The grid is made of pyrolytic carbon and ceramic insulators are used as an isolator between the three grids. The gap between the grids is $0.5 \mathrm{~mm}$ and the ion beam diameter is $12 \mathrm{~mm}$. The screen grid and ion source were biased to $+1,200 \mathrm{~V}$ with respect to ground and the acceleration grid was set to $-200 \mathrm{~V}$. The extracted ion beam was estimated as the current through the screen power supply minus the current through the accelerator power supply. The validity of this method was shown in our previous study ${ }^{8)}$ A neutralizer was not used in this study, as there is little difference between the extracted ion beam current without a neutralizer and that with a filament neutralizer $(\phi=0.2$ $\mathrm{mm} \times 100 \mathrm{~mm}, 2 \%$ thoriated tungsten ${ }^{9}$ ).

In this study, krypton, rather than xenon, is used as a propellant gas. A previous study showed that the laser induced some perturbation on the xenon plasma ${ }^{10)}$. This is due to photo-ionization of excited xenon atoms; xenon atoms at the excited state (ionization potential $12.1 \mathrm{eV}$ ) are ionized by the laser (wavelength $532 \mathrm{~nm}$ and photon energy $2.3 \mathrm{eV}$ ), while no effect is observed for krypton plasma. A thermal mass flow controller was used. The flow rate error is less than $5 \%$ for most of the conditions. A $0.3 \mathrm{~m}$ diameter by $0.4 \mathrm{~m}$ long vacuum chamber was used in the experiments. The pumping system comprised a turbo molecular pump. The background pressure was maintained below $5.0 \times 10^{-2} \mathrm{~Pa}$ for most of the operating conditions.

\subsection{LTS setup}

Figure 2 shows the experimental setup for LTS measurements on the miniature microwave discharge ion thruster. The scattering light inside the discharge chamber is collected through a hole $(\phi=3 \mathrm{~mm})$ and the laser beam go pass through two small holes $(\phi=2 \mathrm{~mm})$. The $\phi=3 \mathrm{~mm}$ hole is positioned at an angle of 90 degree to the laser pass.

The light source is the second harmonic beam of an $\mathrm{Nd}$ :YAG laser having a wavelength of $532 \mathrm{~nm}$ with an energy of $180 \mathrm{~mJ}$, a repetition rate of $10 \mathrm{~Hz}$, a pulse width of $6 \mathrm{~ns}$ and a beam divergence of $0.6 \mathrm{mrad}$. The measurement point is 2 $\mathrm{mm}$ upstream of the screen grid on the thruster axis ( $z$ axis). The laser beam is focused through a focusing lens $(f=300 \mathrm{~mm})$. The size of the focal spot was estimated as $80 \mu \mathrm{m}$ in diameter, by observing the spatial profile of Rayleigh scattering from 40 $\mathrm{kPa}$ air gas. Scattered light from the plasma is focused onto the entrance slit of the triple grating spectrometer (TGS) with two achromatic lenses of $f=350 \mathrm{~mm}$ and $f=250 \mathrm{~mm}$. The slit width is $200 \mu \mathrm{m}$. The scattering volume is $0.08 \times 0.08 \times 2 \mathrm{~mm}^{3}$, as determined by the laser beam size, the slit width and the slit height, respectively.

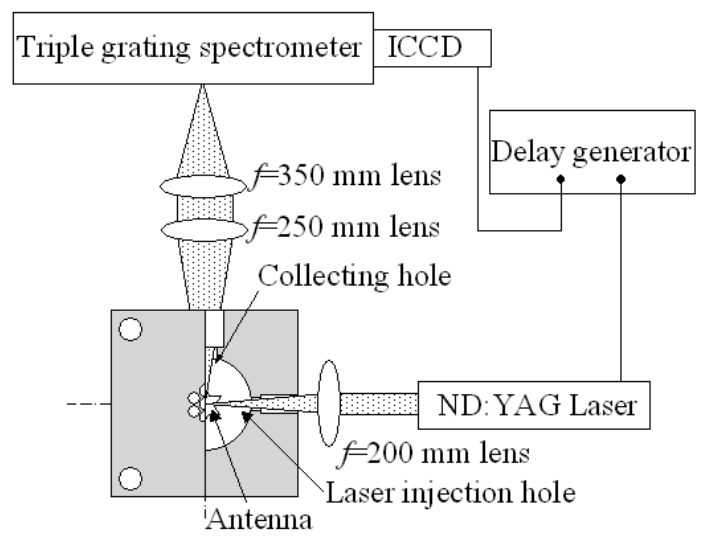

(a)

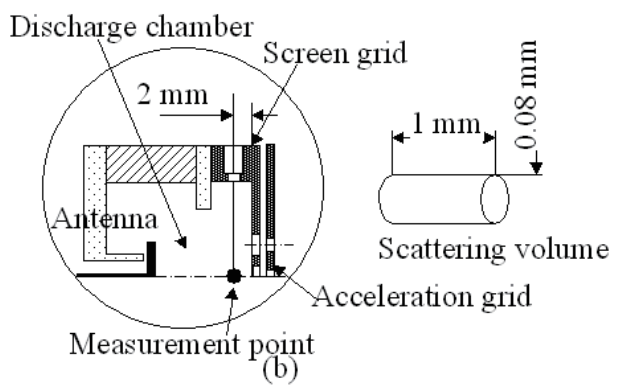

Fig. 2. Schematic diagram of LTS measurements system on the 
miniature microwave discharge ion thruster. (a) front view (b) side view The solid angle of observation is about 0.025 sr. Strong stray light is generated from the surface of the components, due to the small size of discharge chamber, and the LTS signals are overwhelmed by it. In order to reduce stray light, the discharge chamber wall was made of carbon and TGS was used. The scattered light is dispersed by passing through the TGS, and is detected by an Intensified Charge Coupled Device (ICCD) camera. The TGS used in this experiment could reduce stray light around $10^{-8}$ at the wavelength of $2 \mathrm{~nm}$ from the probing laser, where the LTS signal is observed.

The estimated Thomson scattered photon number is so small that we used a photon counting method. The detected Thomson scattered signals were analyzed by a photon counting mode after more than 5000 laser shots had been accumulated. The data accumulation process technique, taking advantage of the DC or repetitive operation of some discharges, was first suggested for lowering the limiting electron densities by Sakoda et al. ${ }^{11)}$. We count photons for two conditions at each condition, condition 1 ; with plasma and laser and condition 2; plasma without laser. We also count photons for the condition 3; laser without plasma. We evaluate actual LTS signal by subtracting the number of photons obtained for condition 2 and 3 from the number of photons obtained for condition 1 .

\section{Results and Discussion}

Figure 3 shows the measured Thomson scattering spectrum at $P_{\mathrm{i}}=8 \mathrm{~W}, \dot{m}=6.2 \mu \mathrm{g} / \mathrm{s}$ and $V_{\mathrm{b}}=1200 \mathrm{~V}$ and $V_{\mathrm{a}}=-200 \mathrm{~V}$. The center of the profile was cut by Rayleigh block, which eliminate the strong stray light. From the shape of the Thomson spectrum, we conclude that the electron energy distribution function was Maxwellian. This result shows flux tube model can be used to simulate ion beam trajectory for the ion engine grid erosion evaluation code; calculation cost is much lower than that by Full Particle in Cell (PIC) model. Because Miyasaka et. $\mathrm{al}^{12)}$ shows there is no difference between the numerical results using full-Particle In Cell (PIC) model $^{13)}$ and that using flux tube model ${ }^{14,15)}$, if EEDF in the vicinity of the screen grid is Maxwellian.

From this spectrum and the Rayleigh scattering calibration using nitrogen gas, $N_{\mathrm{e}}$ and $T_{\mathrm{e}}$ were calculated to be $(3.8 \pm 0.2) \times 10^{17} \mathrm{~m}-3$ and $6.2 \pm 0.1 \mathrm{eV}$, respectively. The experimental uncertainty for each point was determined primarily by the statistical fluctuation in the number of detected photons ${ }^{16)}$. The ion saturation current from this result is estimated as $4.9 \mathrm{~mA}$, which is equal to the ion beam current through the screen grid of $4.9 \mathrm{~mA}$.

Ion saturation current was estimated as follows. According to the Bohm sheath criterion, the velocity of an ion from the plasma into the ion sheath on a grid is assumed to be Bohm velocity, $V_{\mathrm{B}}$, expressed as follows.

$$
V_{B}=\sqrt{\frac{k T_{e}}{M_{i}}}
$$

Given that $N_{\mathrm{e}}$ in the bulk plasma decreases by a factor of exp $(-1 / 2)$ at the point where the ion velocity reaches $V_{\mathrm{B}}$, the ion beam saturation current is evaluated as below.

$$
I_{\mathrm{b}}=e N_{\mathrm{e}} \exp \left(-\frac{1}{2}\right) S \sqrt{\frac{k T_{\mathrm{e}}}{M_{\mathrm{i}}}}
$$

The good agreement of ion saturation current with the ion beam current through screen grid shows that LTS technique is useful and tool for the measurement of plasma property.

Figure 4 shows the relation between plasma parameters (electron number density and temperature) and incident microwave power at $\dot{m}=9.3 \mu \mathrm{g} / \mathrm{s}$ and $V_{\mathrm{b}}=1200 \mathrm{~V}$ and $V_{\mathrm{a}}=$ $-200 \mathrm{~V}$. The number density is linearly increased with incident microwave power; the electron temperature is almost constant. The increase in $N_{\mathrm{e}}$ at the vicinity of the screen grid would be due to the increase in $N_{\mathrm{e}}$ in the region between magnetic mirrors (between the front yoke and the central yoke), where ionization occurs ${ }^{17)}$. On the other hand, electrons lose energy while diffusion from the ionization region to the measurement point, so the electron temperature at the measurement point remains almost constant. Therefore, the ion beam current increase with increase in $P_{\mathrm{i}}{ }^{18)}$.

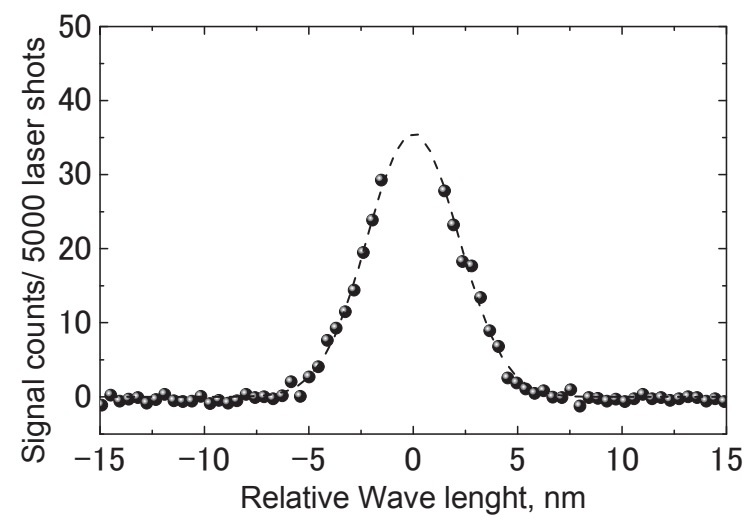

Fig. 3. Spectrum of Thomson scattering.

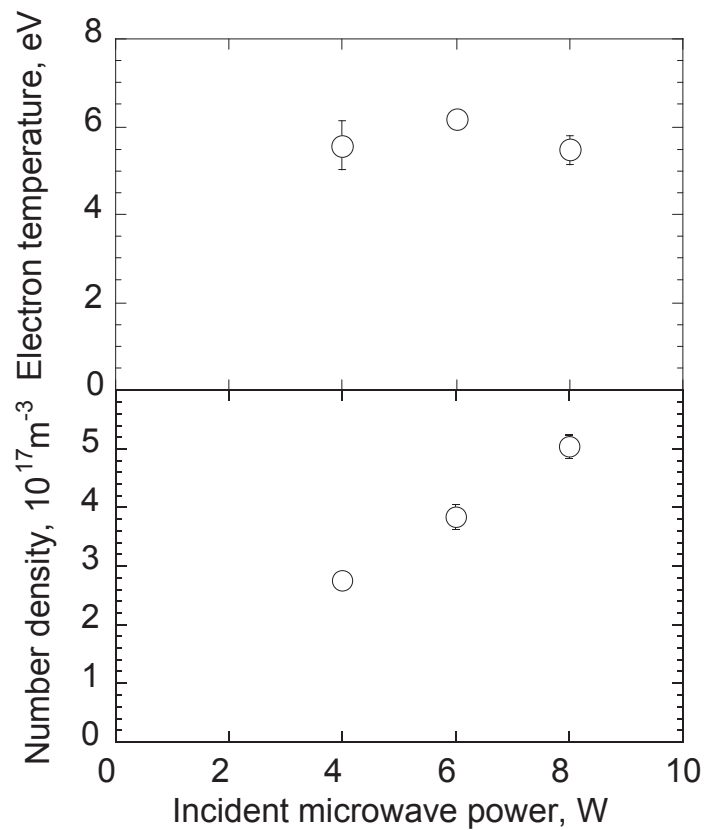

Fig. 4. Dependence of plasma parameters on incident microwave power. 


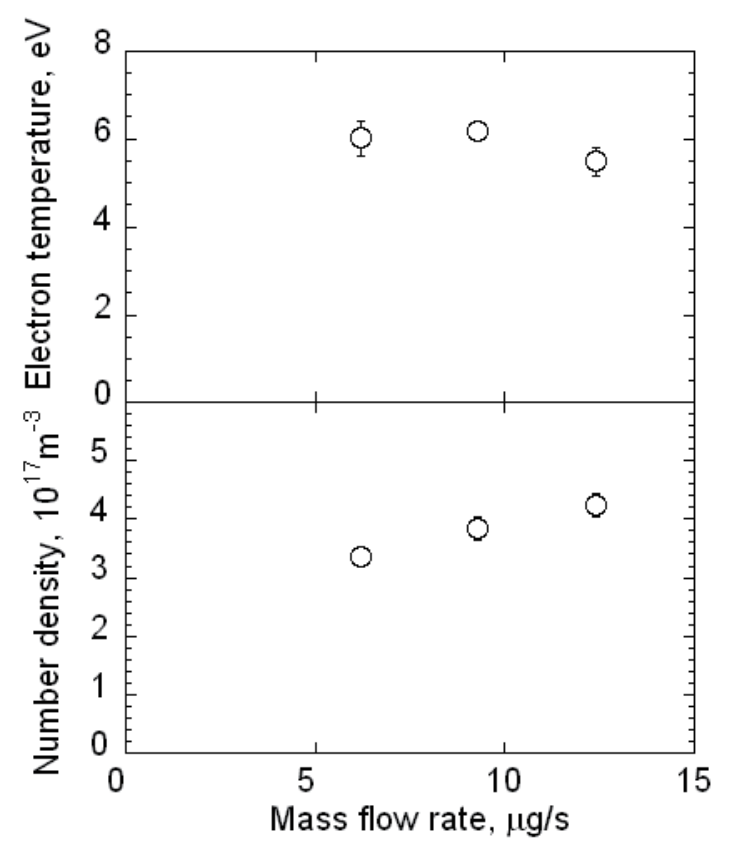

Fig. 5. Dependence of plasma parameters on mass flow rate.

Figure 5 shows the dependence of plasma parameters (electron number density and temperature) on mass flow rate at $P_{\mathrm{i}}=6 \mathrm{~W}$ and $V_{\mathrm{b}}=1200 \mathrm{~V}$ and $V_{\mathrm{a}}=-200 \mathrm{~V}$. The number density increase with $\dot{m}$; the electron temperature is slightly decreased with increase in $\dot{m}$. The number density is proportional to $\left\{1-\exp \left[\alpha\left(1-\eta_{\mathrm{u}}\right) \dot{m}\right]\right\}$, rather than $\dot{m}$, where $\alpha$ is a constant. This is because the chance of the ionization is increased with the number density of neutral atoms in the discharge chamber; the neutral atom number density is proportional to pressure inside the discharge chamber and this is proportional to $\left\{1-\exp \left[\alpha\left(1-\eta_{\mathrm{u}}\right) \dot{m}\right]\right\}$. The increase in $N_{\mathrm{e}}$ at the vicinity of the screen grid would be due to the increase in $N_{\mathrm{e}}$ in the discharge chamber; $N_{\mathrm{e}}$ in the discharge chamber with increase in $\dot{m}$ is due to the increase in pressure in the discharge chamber ${ }^{17)}$ as above mentioned. Therefore, the ion beam current increase with increase in $\dot{m}^{19)}$.

The reason that $T_{\mathrm{e}}$ is slightly decreased with increase in $\dot{m}$ is that the mean free path of the electrons is shortened with the increase in $\dot{m}$, which leads the increase in the pressure inside the discharge chamber. The decrease in the mean free path leads to the reduction in the chance that electrons will go through the ECR layer while collision; electrons receive less energy from the microwaves and this leads to the decrease in $T_{\mathrm{e}}$.

Table 2 shows ion saturation current deduced from LTS measurement and ion beam current through the screen grid for several conditions. We assumed isotropic EEDF, since measurement point is not sheath region and the magnetic field strength at measurement point is not so strong(less than 10 $\mathrm{mT})$. The estimated ion saturation currents deduced from LTS are in good agreement with ion beam currents through the screen grid on the almost all the conditions. Though the estimated ion saturation current is larger than ion beam current at most of the conditions, it would be due to the lack of uniformity of the plasma property; we assume each ion beam current through the hole is equal, but the current through the center holes would be larger than that through the others. Since the center hole is further from the wall than the other holes, the effect of loss on the wall is less than the others.

\section{Summary}

The LTS technique presented here contributes to improve the accuracy of a lifetime evaluation numerical code. The LTS technique allows non-intrusive, quantitative measurements of plasma number density and electron energy distribution function. In comparison to electrostatic probe diagnostics, the use of LTS technique is attractive since plasma property in the ion thruster with beam extraction can be directly measured without perturbation of the plasma. The demonstrated LTS probes the electron number density and EEDF in the $30 \mathrm{~W}$ class microwave discharge ion thruster. A photon counting method and a triple grating spectrometer were used against a small Thomson scattering signal and a strong stray laser light. As a detector, ICCD camera was used, this contributes to the reduction of the measurement period; the measurement period is $1 / 10$ times less than that using a photomultiplier tube as a detector.

The observed Thomson scattering spectra tell the EEDF of the plasma in the vicinity of the screen grid is Maxwellian. These results ensure that flux tube model can be used for ion beam trajectory simulation for the ion engine grid erosion evaluation code.

The estimated ion saturation currents are in good agreement with the ion beam currents through screen grid holes. These results show that LTS technique is useful and validate tool for the measurement of plasma property and contribute to improve the accuracy of erosion evaluation code.

We have made a study on the dependence of plasma property on incident microwave power and found a relatively linear dependence. We also investigated the dependence of the number density on mass flow rate. The dependencies of the plasma parameter on operational parameters are useful to design the grid system of the ion thruster.

Table 2. Ion saturation current vs. ion beam current for several conditions.

\begin{tabular}{ccccc}
\hline \hline $\begin{array}{c}\text { Incident microwave } \\
\text { power, W }\end{array}$ & Mass flow rate, $\mu \mathrm{g} / \mathrm{s}$ & $\begin{array}{c}\text { Ion saturation current } \\
\text { (from LTS), } \mathrm{mA}\end{array}$ & $\begin{array}{c}\text { Extracted screen } \\
\text { current, } \mathrm{mA}\end{array}$ & $\begin{array}{c}\text { Acceleration } \\
\text { current, } \mathrm{mA}\end{array}$ \\
\hline 4 & 9.3 & 3.4 & 3.1 & 0.1 \\
6 & 9.3 & 5.0 & 4.7 & 1 \\
8 & 9.3 & 6.1 & 5.6 & 1.9 \\
6 & 6.2 & 4.3 & 4.4 & 0.8 \\
6 & 12 & 4.9 & 4.7 & 0.9 \\
\hline
\end{tabular}




\section{Acknowledgments}

This work was supported by Kyushu University Interdisciplinary Programs in Education and Projects in Research Development, the Engineering Digital Innovation Center and the Institute of Space and Astronautical Science of the Japan Aerospace Exploration Agency and the Japan Society for the Promotion of Science, Japan for their financial support through a Grant-in-Aid for Young Scientists (A), No. 23686123. The authors also thank Prof. Masakatsu Nakano for useful comments.

\section{References}

1) Nakano, M.: Grid Erosion Analysis and Lifetime Evaluation of Ion Engines, Frontier of Applied Plasma Technology, 2 (2009), pp.21-24.

2) Herman, D. A. and Gallimore, A. D.: An ion thruster internal discharge chamber electrostatic probe diagnostic technique using a high-speed probe positioning system, Rev. Sci. Instrument, 79 (2008), 013302.

3) Sheffield, J., Plasma Scattering of Electromagnetic Radiation, Academic press, New York, 1975, Chap. 9.

4) Evans, D. E., and Katzenstein, J.: Laser Light Scattering in Laboratory Plasmas, Report on Progress in Physics, 32 (1969), pp. 207-271.

5) Muraoka, K., Uchino, K., and Bowden, M. D.: Diagnostics of Low-density Glow Discharge Plasmas Using Thomson Scattering, Plasma Physics and Controlled Fusion, 40 (1998), pp.1221-1239.

6) Yamamoto, N., Masui, H., Kataharada, H., Nakashima, H., and Takao, Y.: Antenna Configuration Effects on Thrust Performance of Miniature Microwave Discharge Ion Engine, Journal of Propulsion and Power, 22 ( 2006), pp. 925-928.

7) Nakano, M., Tachibana, T., and Arakawa, Y. : A Scaling Law of the Life Estimation of the Three-Grid Optics for an Ion Engin, Transactions of the Japan Society for Aeronautical and Space Sciences, 45 (2002), pp.154-161.

8) Kataharada, H., Takao, Y., Yamamoto, N., Ijiri, H. and Nakashima, H.: Development of Small Microwave Discharge Ion Thruster, Thin Solid Films, 506 (2006), pp. 605-608.

9) Takao, Y., Kataharada, H., Miyamoto, T., Masui, H., Yamamoto, N. and Nakashima, H.: Performance Test of Micro Ion Thruster using Microwave Discharge, Vacuum, 80 (2006), pp.1239-1243.

10) Kim, Y. K., Tomita, K., Hassaballa, S., Uchino, K., Muraoka, K., Hatanaka, H., Kim, Y. M., Lee, S. E., Son, S. H. and Jang, S. H.: Development of an Infrared Laser Thomson Scattering System for Measurements of Electron Density and Electron Temperature of a PDP Micro-discharge Plasma, SID International Symposium Digest of Technical Papers, 35 (2004), pp. 550-553.

11) Sakoda, T., Momii, S. Uchino K., Muraoka, K., Bowden, M., Maeda, M., Manabe, Y., Kitagawa, M., and Kimura, T.: Thomson Scattering diagnostics of an ECR Processing Plasma, Jpn. J. Appl. Phys., 30 (1991), pp.L1425-L1427.

12) Miyasaka, T., Kobayashi, T. and Asato, K.: Numerical Prediction of Grid Erosion of Ion Engine, Transactions of the Japanese Society for Artificial Intelligence, Aerospace Technology Japan, $\mathbf{8}$ (2011), pp. Pb_61-Pb_66.

13) Vahedi, V. and Surendra, M.: A Monte Carlo Collision Model for the Particle-in-cell Method: Applications to Argon and Oxygen Discharges, Computer Physics Communications, 87 (1995), pp179-198

14) Nakayama, Y. and Wilbur, P. J.: Numerical Simulation of Ion Beam Optics for Multiple-Grid Systems, Journal of Propulsion and Power, 19 (2003), pp.607-613.

15) Arakawa, Y. and Ishihara, K.: A Numerical Code for Cusped Ion Thruster, Proceedings of 22nd International Electric Propulsion Conference, IEPC-91-118, 1991.

16) Kunze, H. J.: The laser as a tool for plasma diagnostic, Plasma diagnostics, ed. Lochte-Holtgreven W, Noth-Holland Publishing Company, Amsterdam (1968), 550.

17) Yamamoto, N., Tomita, K., Yamasaki, N., Tsuru, T., Ezaki, T., Kotani, Y., Uchino K. and Nakashima, H.: Measurements of Electron Density and Temperature in a Miniature Microwave Discharge Ion Thruster Using Laser Thomson Scattering Technique, Plasma Sources Science and Technology, 19 (2010), 045009.

18) Masui, H., Tashiro, Y., Yamamoto, N., Nakashima, H. and Funaki, I.: Analysis of Electron and Microwave Behaviors in Microwave Discharge Neutralizer, Trans. Jpn. Society for Aeronautical and Space Sciences, 49 (2006), pp.87-93.

19) Yamamoto, N., Kondo, S., Chikaoka, T., Masui H. and Nakashima, H.: Effects of Magnetic Field Configuration on Thrust Performance in A Miniature Microwave Discharge Ion Thruster, Journal of Applied Physics, 102 ( 2007), 123304. 